\title{
On the connections between Pell numbers and Fibonacci $p$-numbers
}

\author{
Anthony G. Shannon ${ }^{1}$, Özgür Erdağ ${ }^{2}$ and Ömür Deveci ${ }^{3}$ \\ ${ }^{1}$ Warrane College, University of New South Wales \\ Kensington, Australia \\ e-mail: tshannon38@gmail.com \\ ${ }^{2}$ Department of Mathematics, Faculty of Science and Letters \\ Kafkas University 36100, Turkey \\ e-mail: ozgur_erdag@hotmail.com \\ ${ }^{3}$ Department of Mathematics, Faculty of Science and Letters \\ Kafkas University 36100, Turkey \\ e-mail: odeveci36@hotmail.com
}

Received: 24 April 2020

Revised: 4 January 2021

Accepted: 7 January 2021

\begin{abstract}
In this paper, we define the Fibonacci-Pell $p$-sequence and then we discuss the connection of the Fibonacci-Pell $p$-sequence with the Pell and Fibonacci $p$-sequences. Also, we provide a new Binet formula and a new combinatorial representation of the Fibonacci-Pell $p$-numbers by the aid of the $n$-th power of the generating matrix of the Fibonacci-Pell $p$-sequence. Furthermore, we derive relationships between the Fibonacci-Pell $p$-numbers and their permanent, determinant and sums of certain matrices.
\end{abstract}

Keywords: Pell sequence, Fibonacci $p$-sequence, Matrix, Representation.

2010 Mathematics Subject Classification: 11K31, 11C20, 15A15.

\section{Introduction}

The well-known Pell sequence $\left\{P_{n}\right\}$ is defined by the following recurrence relation:

$$
P_{n+2}=2 P_{n+1}+P_{n} \text { for } n \geq 0 \text { in which } P_{0}=0 \text { and } P_{1}=1 \text {. }
$$


There are many important generalizations of the Fibonacci sequence. The Fibonacci $p$-sequence $[22,23]$ is one of them:

$$
F_{p}(n)=F_{p}(n-1)+F_{p}(n-p-1) \text { for } p=1,2,3, \ldots \text { and } n>p
$$

in which $F_{p}(0)=0, F_{p}(1)=\cdots=F_{p}(p)=1$. When $p=1$, the Fibonacci $p$-sequence $\left\{F_{p}(n)\right\}$ is reduced to the usual Fibonacci sequence $\left\{F_{n}\right\}$.

It is easy to see that the characteristic polynomials of the Pell sequence and Fibonacci $p$-sequence are $f_{1}(x)=x^{2}-2 x-1$ and $f_{2}(x)=x^{p+1}-x^{p}-1$, respectively. We use these in the next section.

Let the $(n+k)$-th term of a sequence be defined recursively by a linear combination of the preceding $k$ terms:

$$
a_{n+k}=c_{0} a_{n}+c_{1} a_{n+1}+\cdots+c_{k-1} a_{n+k-1},
$$

in which $c_{0}, c_{1}, \ldots, c_{k-1}$ are real constants. In [12], Kalman derived a number of closed-form formulas for the generalized sequence by the companion matrix method as follows:

Let the matrix $A$ be defined by

$$
A=\left[a_{i, j}\right]_{k \times k}=\left[\begin{array}{cccccc}
0 & 1 & 0 & \cdots & 0 & 0 \\
0 & 0 & 1 & \cdots & 0 & 0 \\
0 & 0 & 0 & \cdots & 0 & 0 \\
\vdots & \vdots & \vdots & \ddots & \vdots & \vdots \\
0 & 0 & 0 & \cdots & 0 & 1 \\
c_{0} & c_{1} & c_{2} & \cdots & c_{k-2} & c_{k-1}
\end{array}\right]
$$

then

$$
A^{n}\left[\begin{array}{c}
a_{0} \\
a_{1} \\
\vdots \\
a_{k-1}
\end{array}\right]=\left[\begin{array}{c}
a_{n} \\
a_{n+1} \\
\vdots \\
a_{n+k-1}
\end{array}\right]
$$

for $n \geq 0$.

Several authors have used homogeneous linear recurrence relations to deduce miscellaneous properties for a plethora of sequences: see for example, [1,4,8-11,19-21,24]. In [5-7, 14-16,22, $23,25]$, the authors defined some linear recurrence sequences and gave their various properties by matrix methods.

In the present paper, we discuss connections between the Pell and Fibonacci $p$-numbers. Firstly, we define the Fibonacci-Pell $p$-sequence and then we study recurrence relation among this sequence, Pell and Fibonacci $p$-sequences. In addition, we obtain their generating matrices, Binet formulas, permanental, determinantal, combinatorial, exponential representations, and we derive a formula for the sums of the Fibonacci-Pell $p$-numbers. 


\section{Main results}

Now we define the Fibonacci-Pell $p$-sequence $\left\{F_{n}^{P, p}\right\}$ by the following homogeneous linear recurrence relation for any given $p(3,4,5, \ldots)$ and $n \geq 0$

$$
F_{n+p+3}^{P, p}=3 F_{n+p+2}^{P, p}-F_{n+p+1}^{P, p}-F_{n+p}^{P, p}+F_{n+2}^{P, p}-2 F_{n+1}^{P, p}-F_{n}^{P, p},
$$

in which $F_{0}^{P, p}=\cdots=F_{p+1}^{P, p}=0$ and $F_{p+2}^{P, p}=1$.

First, we consider the relationship between the Fibonacci-Pell $p$-sequence which is defined above, Pell, and Fibonacci $p$-sequences.

Theorem 2.1. Let $P_{n}, F_{3}(n)$ and $F_{n}^{P, 3}$ be the $n$-th Pell number, Fibonacci 3-number, and FibonacciPell 3-numbers, respectively. Then, for $n \geq 0$

$$
P_{n+2}=F_{n+5}^{P, 3}+2 F_{n+3}^{P, 3}+F_{3}(n+2)+F_{3}(n) .
$$

Proof. The assertion may be proved by induction on $n$. It is clear that

$$
P_{2}=F_{5}^{P, 3}+2 F_{3}^{P, 3}+F_{3}(2)+F_{3}(0)=2 .
$$

Suppose that the equation holds for $n \geq 1$. Then we must show that the equation holds for $n+1$. Since the characteristic polynomial of Fibonacci-Pell $p$-sequence $\left\{F_{n}^{P, p}\right\}$, is

$$
g(x)=x^{p+3}-3 x^{p+2}+x^{p+1}+x^{p}-x^{2}+2 x+1
$$

and

$$
g(x)=f_{1}(x) f_{2}(x),
$$

where $f_{1}(x)$ and $f_{2}(x)$ are the characteristic polynomials of Pell sequence and Fibonacci $p$-sequence, respectively, we obtain the following relations:

$$
P_{n+6}=3 P_{n+5}-P_{n+4}-P_{n+3}+P_{n+2}-2 P_{n+1}-P_{n}
$$

and

$$
F_{3}(n+6)=3 F_{3}(n+5)-F_{3}(n+4)-F_{3}(n+3)+F_{3}(n+2)-2 F_{3}(n+1)-F_{3}(n)
$$

for $n \geq 1$. Thus, the conclusion is obtained.

Theorem 2.2. Let $P_{n}$ and $F_{n}^{P, p}$ be the $n$-th Pell number and Fibonacci-Pell p-numbers. Then, for $n \geq 0$ and $p \geq 3$.

$i$. Let $p$ be a positive integer, then

$$
P_{n}=F_{n+p+1}^{P, p}-F_{n+p}^{P, p}-F_{n}^{P, p} .
$$

ii. If $p$ is odd, then

$$
P_{n}+P_{n+1}=F_{n+p+2}^{P, p}-F_{n+p}^{P, p}-F_{n+1}^{P, p}-F_{n}^{P, p}
$$

and

iii. If $p$ is odd, then

$$
\sum_{i=0}^{n}\left(F_{i}^{P, p}+P_{i}\right)=F_{n+p+1}^{P, p} .
$$


Proof. Consider the Case ii. The assertion may be proved by induction on $n$. Then for $p=3$, it is clear that $P_{0}+P_{1}=F_{5}^{P, 3}-F_{3}^{P, 3}-F_{1}^{P, 3}-F_{0}^{P, 3}=1$. Suppose that the equation holds for $n>0$. Then we must show that the equation holds for $n+1$. Since the characteristic polynomial of the Pell sequence $\left\{P_{n}\right\}$, is

$$
f_{1}(x)=x^{2}-2 x-1
$$

we obtain the following relations:

$$
P_{n+6}=3 P_{n+5}-P_{n+4}-P_{n+3}+P_{n+2}-2 P_{n+1}-P_{n}
$$

for $n \geq 1$. Now we consider the proof for the case $p>3$. Suppose that the equation holds for $p=2 \alpha+1,(\alpha \in \mathbb{N})$ and $n \geq 0$, it is clear that

$$
P_{n}+P_{n+1}=F_{n+2 \alpha+3}^{P, 2 \alpha+1}-F_{n+2 \alpha+1}^{P, 2 \alpha+1}-F_{n+1}^{P, 2 \alpha+1}-F_{n}^{P, 2 \alpha+1} .
$$

Then we must show that the equation holds for $p=2 \alpha+3,(\alpha \in \mathbb{N})$. For $n=0$, it is clear that

$$
P_{0}+P_{1}=F_{2 \alpha+5}^{P, 2 \alpha+1}-F_{2 \alpha+3}^{P, 2 \alpha+1}-F_{1}^{P, 2 \alpha+1}-F_{0}^{P, 2 \alpha+1}=1 .
$$

The assertion may be proved again by induction on $n$. Assume that the equation holds for $n>0$. Then we must show that the equation holds for $n+1$. Since the characteristic polynomial of the Pell sequence $\left\{P_{n}\right\}$, is

$$
f_{1}(x)=x^{2}-2 x-1
$$

we obtain the following relations:

$$
P_{n+2 \alpha+6}=3 P_{n+2 \alpha+5}-P_{n+2 \alpha+4}-P_{n+2 \alpha+3}+P_{n+2}-2 P_{n+1}-P_{n}
$$

for $n \geq 1$. Thus, the conclusion is obtained.

There is a similar proof for Case $\mathrm{i}$ and Case iii.

By the recurrence relation (1), we have

$$
\left[\begin{array}{c}
F_{n+p+3}^{P, p} \\
F_{n+p+2}^{P, p} \\
F_{n+p+1}^{P, p} \\
\vdots \\
F_{n+1}^{P, p}
\end{array}\right]=\left[\begin{array}{cccccccccc}
3 & -1 & -1 & 0 & \cdots & 0 & 0 & 1 & -2 & -1 \\
1 & 0 & 0 & 0 & \cdots & 0 & 0 & 0 & 0 & 0 \\
0 & 1 & 0 & 0 & \cdots & 0 & 0 & 0 & 0 & 0 \\
0 & 0 & 1 & 0 & \cdots & 0 & 0 & 0 & 0 & 0 \\
0 & 0 & 0 & 1 & \cdots & 0 & 0 & 0 & 0 & 0 \\
\vdots & \vdots & \vdots & \vdots & \ddots & \vdots & \vdots & \vdots & \vdots & \vdots \\
0 & 0 & 0 & 0 & \cdots & 1 & 0 & 0 & 0 & 0 \\
0 & 0 & 0 & 0 & \cdots & 0 & 1 & 0 & 0 & 0 \\
0 & 0 & 0 & 0 & \cdots & 0 & 0 & 1 & 0 & 0 \\
0 & 0 & 0 & 0 & \cdots & 0 & 0 & 0 & 1 & 0
\end{array}\right]\left[\begin{array}{c}
F_{n+p+2}^{P, p} \\
F_{n+p+1}^{P, p} \\
F_{n+p}^{P, p} \\
\vdots \\
F_{n}^{P, p}
\end{array}\right]
$$

for the Fibonacci-Pell $p$-sequence $\left\{F_{n}^{P, p}\right\}$. Letting 


$$
D_{p}=\left[\begin{array}{cccccccccc}
3 & -1 & -1 & 0 & \cdots & 0 & 0 & 1 & -2 & -1 \\
1 & 0 & 0 & 0 & \cdots & 0 & 0 & 0 & 0 & 0 \\
0 & 1 & 0 & 0 & \cdots & 0 & 0 & 0 & 0 & 0 \\
0 & 0 & 1 & 0 & \cdots & 0 & 0 & 0 & 0 & 0 \\
0 & 0 & 0 & 1 & \cdots & 0 & 0 & 0 & 0 & 0 \\
\vdots & \vdots & \vdots & \vdots & \ddots & \vdots & \vdots & \vdots & \vdots & \vdots \\
0 & 0 & 0 & 0 & \cdots & 1 & 0 & 0 & 0 & 0 \\
0 & 0 & 0 & 0 & \cdots & 0 & 1 & 0 & 0 & 0 \\
0 & 0 & 0 & 0 & \cdots & 0 & 0 & 1 & 0 & 0 \\
0 & 0 & 0 & 0 & \cdots & 0 & 0 & 0 & 1 & 0
\end{array}\right]_{(p+3) \times(p+3)}
$$

the companion matrix $D_{p}=\left[d_{i, j}\right]_{(p+3) \times(p+3)}$ is said to be the Fibonacci-Pell $p$-matrix. For more details on the companion type matrices, see $[17,18]$. It can be readily established by mathematical induction that for $p \geq 3$ and $n \geq 3 p-1$,

$$
\left(D_{p}\right)^{n}=\left[\begin{array}{cccccc}
F_{n+p+2}^{P, p} & F_{p}(n-p+1)-F_{n+p+1}^{P, p}-F_{n+p}^{P, p} & F_{p}(n-p+2)-F_{n+p+1}^{P, p} & F_{p}(n-p+3) & \cdots & \\
F_{n+p+1}^{P, p} & F_{p}(n-p)-F_{n+p}^{P, p}-F_{n+p-1}^{P, p} & F_{p}(n-p+1)-F_{n+p}^{P, p} & F_{p}(n-p+2) & \cdots & \\
F_{n+p}^{P, p} & F_{p}(n-p-1)-F_{n+p-1}^{P, p}-F_{n+p-2}^{P, p} & F_{p}(n-p)-F_{n+p-1}^{P, p} & F_{p}(n-p+1) & \cdots & D_{p}^{*} \\
\vdots & \vdots & \vdots & \vdots & \ddots & \\
F_{n+1}^{P, p} & F_{p}(n-2 p)-F_{n}^{P, p}-F_{n-1}^{P, p} & F_{p}(n-2 p+1)-F_{n}^{P, p} & F_{p}(n-2 p+2) & \cdots & \\
F_{n}^{P, p} & F_{p}(n-2 p-1)-F_{n-1}^{P, p}-F_{n-2}^{P, p} & F_{p}(n-2 p)-F_{n-1}^{P, p} & F_{p}(n-2 p+1) & \cdots &
\end{array}\right],
$$

where

$$
D_{p}^{*}=\left[\begin{array}{ccc}
F_{p}(n) & F_{p}(n-p+3)+F_{p}(n-p)+F_{p}(n-p-1)+\cdots+F_{p}(n-2 p+3)-F_{n+p+2}^{P, p} & -F_{n+p+1}^{P, p} \\
F_{p}(n-1) & F_{p}(n-p+2)+F_{p}(n-p-1)+F_{p}(n-p-2)+\cdots+F_{p}(n-2 p+2)-F_{n+p+1}^{P, p} & -F_{n+p}^{P, p} \\
F_{p}(n-2) & F_{p}(n-p+1)+F_{p}(n-p-2)+F_{p}(n-p-3)+\cdots+F_{p}(n-2 p+1)-F_{n+p}^{P, p} & -F_{n+p-1}^{P, p} \\
\vdots & \vdots & \vdots \\
F_{p}(n-p-1) & F_{p}(n-2 p+2)+F_{p}(n-2 p-1)+F_{p}(n-2 p-2)+\cdots+F_{p}(n-3 p+2)-F_{n+1}^{P, p} & -F_{n}^{P, p} \\
F_{p}(n-p-2) & F_{p}(n-2 p+1)+F_{p}(n-2 p-2)+F_{p}(n-2 p-3)+\cdots+F_{p}(n-3 p+1)-F_{n}^{P, p} & -F_{n-1}^{P, p}
\end{array}\right]
$$

In [22], Stakhov defined the generalized Fibonacci $p$-matrix $Q_{p}$ and derived the $n$-th power of the matrix $Q_{p}$. In [13], Kilic gave a Binet formula for the Fibonacci $p$-numbers by matrix method. Now we concentrate on finding another Binet formula for the Fibonacci-Pell $p$-numbers by the aid of the matrix $\left(D_{p}\right)^{n}$.

Lemma 2.3. The characteristic equation of all the Fibonacci-Pell p-numbers

$$
x^{p+3}-3 x^{p+2}+x^{p+1}+x^{p}-x^{2}+2 x+1=0
$$

does not have multiple roots for $p \geq 3$. 
Proof. It is clear that $x^{p+3}-3 x^{p+2}+x^{p+1}+x^{p}-x^{2}+2 x+1=\left(x^{p+1}-x^{p}-1\right)\left(x^{2}-2 x-1\right)$. In [13], it was shown that the equation $x^{p+1}-x^{p}-1=0$ does not have multiple roots for $p>1$. It is easy to see that the roots of the equation $x^{2}-2 x-1=0$ are $1+\sqrt{2}$ and $1-\sqrt{2}$. Since $(1+\sqrt{2})^{p+1}-(1+\sqrt{2})^{p}-1 \neq 0$ and $(1-\sqrt{2})^{p+1}-(1-\sqrt{2})^{p}-1 \neq 0$, the equation $x^{p+3}-3 x^{p+2}+x^{p+1}+x^{p}-x^{2}+2 x+1=0$ does not have multiple roots for $p \geq 3$.

Let $\alpha_{1}, \alpha_{2}, \ldots, \alpha_{p+3}$ be the roots of the equation $x^{p+3}-3 x^{p+2}+x^{p+1}+x^{p}-x^{2}+2 x+1=0$ and let $V_{p}$ be a $(p+3) \times(p+3)$ Vandermonde matrix as follows:

$$
V_{p}=\left[\begin{array}{cccc}
\left(\alpha_{1}\right)^{p+2} & \left(\alpha_{2}\right)^{p+2} & \ldots & \left(\alpha_{p+3}\right)^{p+2} \\
\left(\alpha_{1}\right)^{p+1} & \left(\alpha_{2}\right)^{p+1} & \ldots & \left(\alpha_{p+3}\right)^{p+1} \\
\vdots & \vdots & \ddots & \vdots \\
\alpha_{1} & \alpha_{2} & \ldots & \alpha_{p+3} \\
1 & 1 & \cdots & 1
\end{array}\right]
$$

Assume that $V_{p}(i, j)$ is a $(p+3) \times(p+3)$ matrix derived from the Vandermonde matrix $V_{p}$ by replacing the $j$-th column of $V_{p}$ by $W_{p}(i)$, where, $W_{p}(i)$ is a $(p+3) \times 1$ matrix as follows:

$$
W_{p}(i)=\left[\begin{array}{c}
\left(\alpha_{1}\right)^{n+p+3-i} \\
\left(\alpha_{2}\right)^{n+p+3-i} \\
\vdots \\
\left(\alpha_{p+3}\right)^{n+p+3-i}
\end{array}\right]
$$

Theorem 2.4. Let $p$ be a positive integer such that $p \geq 3$ and let $\left(D_{p}\right)^{n}=d_{i, j}^{(p, n)}$ for $n \geq 1$, then

$$
d_{i, j}^{(p, n)}=\frac{\operatorname{det} V_{p}(i, j)}{\operatorname{det} V_{p}} .
$$

Proof. Since the equation $x^{p+3}-3 x^{p+2}+x^{p+1}+x^{p}-x^{2}+2 x+1=0$ does not have multiple roots for $p \geq 3$, the eigenvalues of the Fibonacci-Pell $p$-matrix $D_{p}$ are distinct. Then, it is clear that $D_{p}$ is diagonalizable. Let $A_{p}=\operatorname{diag}\left(\alpha_{1}, \alpha_{2}, \ldots, \alpha_{p+3}\right)$, then we may write $D_{p} V_{p}=V_{p} A_{p}$. Since the matrix $V_{p}$ is invertible, we obtain the equation $\left(V_{p}\right)^{-1} D_{p} V_{p}=A_{p}$. Therefore, $D_{p}$ is similar to $A_{p}$; hence, $\left(D_{p}\right)^{n} V_{p}=V_{p}\left(A_{p}\right)^{n}$ for $n \geq 1$. So we have the following linear system of equations:

$$
\left\{\begin{array}{c}
d_{i, 1}^{(p, n)}\left(\alpha_{1}\right)^{p+2}+d_{i, 2}^{(p, n)}\left(\alpha_{1}\right)^{p+1}+\cdots+d_{i, p+3}^{(p, n)}=\left(\alpha_{1}\right)^{n+p+3-i} \\
d_{i, 1}^{(p, n)}\left(\alpha_{2}\right)^{p+2}+d_{i, 2}^{(p, n)}\left(\alpha_{2}\right)^{p+1}+\cdots+d_{i, p+3}^{(p, n)}=\left(\alpha_{2}\right)^{n+p+3-i} \\
\vdots \\
d_{i, 1}^{(p, n)}\left(\alpha_{p+3}\right)^{p+2}+d_{i, 2}^{(p, n)}\left(\alpha_{p+3}\right)^{p+1}+\cdots+d_{i, p+3}^{(p, n)}=\left(\alpha_{p+3}\right)^{n+p+3-i} .
\end{array}\right.
$$

Then we conclude that

$$
d_{i, j}^{(p, n)}=\frac{\operatorname{det} V_{p}(i, j)}{\operatorname{det} V_{p}}
$$

for each $i, j=1,2, \ldots, p+3$. 
Thus by Theorem 2.4 and the matrix $\left(D_{p}\right)^{n}$, we have the following useful result for the Fibonacci-Pell $p$-numbers.

Corollary 2.1. Let $p$ be a positive integer such that $p \geq 3$ and let $F_{n}^{P, p}$ be the $n$-th element of the Fibonacci-Pell p-sequence, then

$$
F_{n}^{P, p}=\frac{\operatorname{det} V_{p}(p+3,1)}{\operatorname{det} V_{p}}
$$

and

$$
F_{n}^{P, p}=-\frac{\operatorname{det} V_{p}(p+2, p+3)}{\operatorname{det} V_{p}}
$$

for $n \geq 1$.

It is easy to see that the generating function of the Fibonacci-Pell $p$-sequence $\left\{F_{n}^{P, p}\right\}$ is as follows:

$$
g(x)=\frac{x^{p+2}}{1-3 x+x^{2}+x^{3}-x^{p+1}+2 x^{p+2}+x^{p+3}},
$$

where $p \geq 3$.

Then we can give an exponential representation for the Fibonacci-Pell $p$-numbers by the aid of the generating function with the following Theorem.

Theorem 2.5. The Fibonacci-Pell p-numbers $\left\{F_{n}^{P, p}\right\}$ have the following exponential representation:

$$
g(x)=x^{p+2} \exp \left(\sum_{i=1}^{\infty} \frac{(x)^{i}}{i}\left(3-x-x^{2}+x^{p}-2 x^{p+1}-x^{p+2}\right)^{i}\right),
$$

where $p \geq 3$.

Proof. Since

$$
\ln g(x)=\ln x^{p+2}-\ln \left(1-3 x+x^{2}+x^{3}-x^{p+1}+2 x^{p+2}+x^{p+3}\right)
$$

and

$$
\begin{aligned}
-\ln \left(1-3 x+x^{2}+x^{3}-x^{p+1}+2 x^{p+2}+x^{p+3}\right)= & -\left[-x\left(3-x-x^{2}+x^{p}-2 x^{p+1}-x^{p+2}\right)-\right. \\
& \frac{1}{2} x^{2}\left(3-x-x^{2}+x^{p}-2 x^{p+1}-x^{p+2}\right)^{2}-\cdots \\
& \left.-\frac{1}{i} x^{i}\left(3-x-x^{2}+x^{p}-2 x^{p+1}-x^{p+2}\right)^{i}-\cdots\right]
\end{aligned}
$$

it is clear that

$$
g(x)=x^{p+2} \exp \left(\sum_{i=1}^{\infty} \frac{(x)^{i}}{i}\left(3-x-x^{2}+x^{p}-2 x^{p+1}-x^{p+2}\right)^{i}\right)
$$

and by a simple calculation, we obtain the conclusion. 
Let $K\left(k_{1}, k_{2}, \ldots, k_{v}\right)$ be a $v \times v$ companion matrix as follows:

$$
K\left(k_{1}, k_{2}, \ldots, k_{v}\right)=\left[\begin{array}{cccc}
k_{1} & k_{2} & \cdots & k_{v} \\
1 & 0 & \cdots & 0 \\
\vdots & \ddots & \ddots & \vdots \\
0 & \cdots & 1 & 0
\end{array}\right]
$$

Theorem 2.6. (Chen and Louck [3]) The $(i, j)$ entry $k_{i, j}^{(n)}\left(k_{1}, k_{2}, \ldots, k_{v}\right)$ in the matrix $K^{n}\left(k_{1}, k_{2}, \ldots, k_{v}\right)$ is given by the following formula:

$$
k_{i, j}^{(n)}\left(k_{1}, k_{2}, \ldots, k_{v}\right)=\sum_{\left(t_{1}, t_{2}, \ldots, t_{v}\right)} \frac{t_{j}+t_{j+1}+\cdots+t_{v}}{t_{1}+t_{2}+\cdots+t_{v}} \times\left(\begin{array}{c}
t_{1}+\cdots+t_{v} \\
t_{1}, \ldots, t_{v}
\end{array}\right) k_{1}^{t_{1}} \cdots k_{v}^{t_{v}},
$$

where the summation is over nonnegative integers satisfying $t_{1}+2 t_{2}+\cdots+v t_{v}=n-i+j$, $\left(\begin{array}{c}t_{1}+\cdots+t_{v} \\ t_{1}, \ldots, t_{v}\end{array}\right)=\frac{\left(t_{1}+\cdots+t_{v}\right) !}{t_{1} ! \cdots t_{v} !}$ is a multinomial coefficient, and the coefficients in (2) are defined to be 1 if $n=i-j$.

Then we can give other combinatorial representations than for the Fibonacci-Pell $p$-numbers by the following Corollary.

Corollary 2.2. Let $F_{n}^{P, p}$ be the $n$-th Fibonacci-Pell $p$-number for $n \geq 1$. Then

$$
F_{n}^{P, p}=\sum_{\left(t_{1}, t_{2}, \ldots, t_{p+3}\right)}\left(\begin{array}{c}
t_{1}+t_{2}+\cdots+t_{p+3} \\
t_{1}, t_{2}, \cdots, t_{p+3}
\end{array}\right) 3^{t_{1}}(-2)^{t_{p+2}}(-1)^{t_{2}+t_{3}+t_{p+3}}
$$

where the summation is over nonnegative integers satisfying

$$
t_{1}+2 t_{2}+\cdots+(p+3) t_{p+3}=n-p-2 .
$$

ii.

$F_{n}^{P, p}=-\sum_{\left(t_{1}, t_{2}, \ldots, t_{p+3}\right)} \frac{t_{p+3}}{t_{1}+t_{2}+\cdots+t_{p+3}} \times\left(\begin{array}{c}t_{1}+t_{2}+\cdots+t_{p+3} \\ t_{1}, t_{2}, \cdots, t_{p+3}\end{array}\right) 3^{t_{1}}(-2)^{t_{p+2}}(-1)^{t_{2}+t_{3}+t_{p+3}}$, where the summation is over nonnegative integers satisfying $t_{1}+2 t_{2}+\cdots+(p+3) t_{p+3}=n+1$. Proof. If we take $i=p+3, j=1$ for the Case i. and $i=p+2, j=p+3$ for the Case ii. in Theorem 2.6, then we can directly see the conclusions from $\left(D_{p}\right)^{n}$.

Now we consider the relationship between the Fibonacci-Pell $p$-numbers and the permanent of a certain matrix which is obtained using the Fibonacci-Pell $p$-matrix $\left(D_{p}\right)^{n}$.

Definition 2.1. A $u \times v$ real matrix $M=\left[m_{i, j}\right]$ is called a contractible matrix in the $k$-th column (respectively, row) if the $k$-th column (respectively, row) contains exactly two non-zero entries.

Suppose that $x_{1}, x_{2}, \ldots, x_{u}$ are row vectors of the matrix $M$. If $M$ is contractible in the $k$-th column such that $m_{i, k} \neq 0, m_{j, k} \neq 0$ and $i \neq j$, then the $(u-1) \times(v-1)$ matrix $M_{i j: k}$ obtained 
from $M$ by replacing the $i$-th row with $m_{i, k} x_{j}+m_{j, k} x_{i}$ and deleting the $j$-th row. The $k$-th column is called the contraction in the $k$-th column relative to the $i$-th row and the $j$-th row.

In [2], Brualdi and Gibson obtained that $\operatorname{per}(M)=\operatorname{per}(N)$ if $M$ is a real matrix of order $\alpha>1$ and $N$ is a contraction of $M$.

Now we concentrate on finding relationships among the Fibonacci-Pell $p$-numbers and the permanents of certain matrices which are obtained by using the generating matrix of the Fibonacci-Pell $p$-numbers. Let $E_{m, p}^{F, P}=\left[e_{i, j}\right]$ be the $m \times m$ super-diagonal matrix, defined by

$$
e_{i, j}=\left\{\begin{array}{cl}
3 \quad & \text { if } i=\tau \text { and } j=\tau \text { for } 1 \leq \tau \leq m, \\
1 \quad & \text { if } i=\tau \text { and } j=\tau+p \text { for } 1 \leq \tau \leq m-p \\
& \text { and } i=\tau+1 \text { and } j=\tau \text { for } 1 \leq \tau \leq m-1, \\
& \text { if } i=\tau \text { and } j=\tau+1 \text { for } 1 \leq \tau \leq m-1, \\
-1 \quad & i=\tau \text { and } j=\tau+2 \text { for } 1 \leq \tau \leq m-2 \\
& \text { and } i=\tau \text { and } j=\tau+p+2 \text { for } 1 \leq \tau \leq m-p-2, \\
-2 \quad & \text { if } i=\tau \text { and } j=\tau+p+1 \text { for } 1 \leq \tau \leq m-p-1, \\
0 & \text { otherwise. }
\end{array}\right.
$$

for $m \geq p+3$. Then we have the following Theorem.

Theorem 2.7. For $m \geq p+3$,

$$
\text { per } E_{m, p}^{F, P}=F_{m+p+2}^{P, p} \text {. }
$$

Proof. Let us consider matrix $E_{m, p}^{F, P}$ and let the equation hold for $m \geq p+3$. Then we show that the equation holds for $m+1$. If we expand the per $E_{m, p}^{F, P}$ by the Laplace expansion of permanent with respect to the first row, then we obtain

per $E_{m+1, p}^{F, P}=3$ per $E_{m, p}^{F, P}-$ per $E_{m-1, p}^{F, P}-$ per $E_{m-2, p}^{F, P}+$ per $E_{m-p, p}^{F, P}-2$ per $E_{m-p-1, p}^{F, P}-$ per $E_{m-p-2, p}^{F, P}$.

Since

$$
\begin{aligned}
\operatorname{per} E_{m, p}^{F, P} & =F_{m+p+2}^{P, p}, \\
\operatorname{per} E_{m-1, p}^{F, P} & =F_{m+p+1}^{P, p}, \\
\operatorname{per} E_{m-2, p}^{F, P} & =F_{m+p}^{P, p}, \\
\operatorname{per} E_{m-p, p}^{F, P} & =F_{m+2}^{P, p}, \\
\operatorname{per} E_{m-p-1, p}^{F, P} & =F_{m+1}^{P, p}, \\
\operatorname{per} E_{m-p-2, p}^{F, P} & =F_{m}^{P, p},
\end{aligned}
$$

we easily obtain that per $E_{m+1, p}^{F, P}=F_{m+p+3}^{P, p}$. So the proof is complete. 
Let $F_{m, p}^{F, P}=\left[f_{i, j}\right]$ be the $m \times m$ matrix, defined by

$$
f_{i, j}=\left\{\begin{array}{cl}
3 \quad & \text { if } i=\tau \text { and } j=\tau \text { for } 1 \leq \tau \leq m-p \\
& \text { if } i=\tau \text { and } j=\tau+p \text { for } 1 \leq \tau \leq m-p \\
1 \quad & i=\tau \text { and } j=\tau \text { for } m-p+1 \leq \tau \leq m \\
& \text { and } i=\tau+1 \text { and } j=\tau \text { for } 1 \leq \tau \leq m-p-1 \\
& \text { if } i=\tau \text { and } j=\tau+1 \text { for } 1 \leq \tau \leq m-p \\
-1 \quad & i=\tau \text { and } j=\tau+2 \text { for } 1 \leq \tau \leq m-p \\
& \text { and } i=\tau \text { and } j=\tau+p+2 \text { for } 1 \leq \tau \leq m-p-2 \\
-2 \quad & \text { if } i=\tau \text { and } j=\tau+p+1 \text { for } 1 \leq \tau \leq m-p-1 \\
0 & \text { otherwise }
\end{array}\right.
$$

for $m \geq p+3$. Then we have the following Theorem.

Theorem 2.8. For $m \geq p+3$,

$$
\text { per } F_{m, p}^{F, P}=F_{m+2}^{P, p} \text {. }
$$

Proof. Let us consider matrix $F_{m, p}^{F, P}$ and let the equation hold for $m \geq p+3$. Then we show that the equation holds for $m+1$. If we expand the per $F_{m, p}^{F, P}$ by the Laplace expansion of permanent with respect to the first row, then we obtain per $F_{m+1, p}^{F, P}=3 \operatorname{per} F_{m, p}^{F, P}-\operatorname{per} F_{m-1, p}^{F, P}-\operatorname{per} F_{m-2, p}^{F, P}+\operatorname{per} F_{m-p, p}^{F, P}-2 \operatorname{per} F_{m-p-1, p}^{F, P}-\operatorname{per} F_{m-p-2, p}^{F, P}$. Since

$$
\begin{gathered}
\text { per } F_{m, p}^{F, P}=F_{m+2}^{P, p}, \\
\operatorname{per} F_{m-1, p}^{F, P}=F_{m+1}^{P, p}, \\
\operatorname{per} F_{m-2, p}^{F, P}=F_{m}^{P, p}, \\
\operatorname{per} F_{m-p, p}^{F, P}=F_{m-p+2}^{P, p}, \\
\operatorname{per} F_{m-p-1, p}^{F, P}=F_{m-p+1}^{P, p}, \\
\operatorname{per} F_{m-p-2, p}^{F, P}=F_{m-p}^{P, p},
\end{gathered}
$$

we easily obtain that per $F_{m+1, p}^{F, P}=F_{m+3}^{P}$. So the proof is complete.

Assume that $G_{m, p}^{F, P}=\left[g_{i, j}\right]$ be the $m \times m$ matrix, defined by

$$
G_{m, p}^{F, P}=\left[\begin{array}{cccccc}
1 & \cdots & 1 & 0 & 0 & 0 \\
1 & & & & & \\
0 & & & \\
\vdots & & & F_{m-1, p}^{F, P} & \\
0 & & & & \\
0 & & &
\end{array}\right] \text {, for } m>p+3
$$

then we have the following results. 
Theorem 2.9. For $m>p+3$,

$$
\operatorname{per} G_{m, p}^{F, P}=\sum_{i=0}^{m+1} F_{i}^{P, p} .
$$

Proof. If we extend per $G_{m, p}^{F, P}$ with respect to the first row, we write

$$
\operatorname{per} G_{m, p}^{F, P}=\operatorname{per} G_{m-1, p}^{F, P}+\operatorname{per} F_{m-1, p}^{F, P} \text {. }
$$

Thus, by the results and an inductive argument, the proof is easily seen.

A matrix $M$ is called convertible if there is an $n \times n(1,-1)$-matrix $K$ such that per $M=\operatorname{det}(M \circ K)$, where $M \circ K$ denotes the Hadamard product of $M$ and $K$.

Now we give relationships among the Fibonacci-Pell $p$-numbers and the determinants of certain matrices which are obtained by using the matrices $E_{m, p}^{F, P}, F_{m, p}^{F, P}$ and $G_{m, p}^{F, P}$. Let $m>p+3$ and let $R$ be the $m \times m$ matrix, defined by

$$
R=\left[\begin{array}{cccccc}
1 & 1 & 1 & \cdots & 1 & 1 \\
-1 & 1 & 1 & \cdots & 1 & 1 \\
1 & -1 & 1 & \cdots & 1 & 1 \\
\vdots & \ddots & \vdots & \ddots & \vdots & \vdots \\
1 & \cdots & 1 & -1 & 1 & 1 \\
1 & \cdots & 1 & 1 & -1 & 1
\end{array}\right]
$$

Corollary 2.3. For $m>p+3$,

$$
\begin{gathered}
\operatorname{det}\left(E_{m, p}^{F, P} \circ R\right)=F_{m+p+2}^{P, p}, \\
\operatorname{det}\left(F_{m, p}^{F, P} \circ R\right)=F_{m+2}^{P, p},
\end{gathered}
$$

and

$$
\operatorname{det}\left(G_{m, p}^{F, P} \circ R\right)=\sum_{i=0}^{m+1} F_{i}^{P, p} .
$$

Proof. Since per $E_{m, p}^{F, P}=\operatorname{det}\left(E_{m, p}^{F, P} \circ R\right)$, per $F_{m, p}^{F, P}=\operatorname{det}\left(F_{m, p}^{F, P} \circ R\right)$ and $\operatorname{per} G_{m, p}^{F, P}$ $=\operatorname{det}\left(G_{m, p}^{F, P} \circ R\right)$ for $m>p+3$, by Theorem 2.7, Theorem 2.8 and Theorem 2.9, we have the conclusion.

Now we consider the sums of the Fibonacci-Pell $p$-numbers. Let

$$
S_{n}=\sum_{i=0}^{n} F_{i}^{P}
$$

for $n \geq 0$ and let $U_{F, P}$ and $\left(U_{F, P}\right)^{n}$ be the $(p+4) \times(p+4)$ matrix such that

$$
U_{F, P}=\left[\begin{array}{cccccc}
1 & 0 & 0 & \cdots & 0 & 0 \\
1 & & & & & \\
0 & & & & \\
\vdots & & & D_{p} & & \\
0 & & & & \\
0 & & & & &
\end{array}\right] .
$$


If we use induction on $n$, then we obtain

$$
\left(U_{F, P}\right)^{n}=\left[\begin{array}{cccccc}
1 & 0 & 0 & \cdots & 0 & 0 \\
S_{n+p+1} & & & & & \\
S_{n+p 4} & & & & & \\
\vdots & & & \left(D_{p}\right)^{n} & & \\
S_{n} & & & & & \\
S_{n-1} & & & & &
\end{array}\right] .
$$

\section{References}

[1] Bradie, B. (2010). Extension and refinements of some properties of sums involving Pell number. Missouri Journal of Mathematical Sciences, 22(1), 37-43.

[2] Brualdi, R. A., \& Gibson, P. M. (1977). Convex polyhedra of doubly stochastic matrices. I. Applications of permanent function, Journal of Combinatorial Theory, Series A, 22(2), 194-230.

[3] Chen, W. Y. C., \& Louck, J. D. (1996). The combinatorial power of the companion matrix. Linear Algebra and its Applications, 232, 261-278.

[4] Devaney, R. L. (1999). The Mandelbrot set, the Farey tree, and the Fibonacci sequence, The American Mathematical Monthly, 106(4), 289-302.

[5] Deveci, O., Adiguzel, Z. \& Dogan, T. (2020). On the Generalized Fibonacci-circulantHurwitz Numbers, Notes on Number Theory and Discrete Mathematics, 26(1), 179-190.

[6] Deveci, O., \& Artun, G. (2019). On the Adjacency-Jacobsthal Numbers. Communications in Algebra, 47 (11), 4520-4532.

[7] Deveci, O., Karaduman, E., \& Campbell, C. M. (2017). The Fibonacci-Circulant Sequences and Their Applications. Iranian Journal of Science and Technology, Transaction A, Science, 41(4), 1033-1038.

[8] Frey, D. D., \& Sellers, J. A. (2000). Jacobsthal numbers and alternating sign matrices. Journal of Integer Sequences, 3, Article 00.2.3.

[9] Gogin, N. D., \& Myllari, A. A. (2007). The Fibonacci-Padovan sequence and MacWilliams transform matrices. Program. Comput. Softw., published in Programmirovanie, 33(2), 74-79.

[10] Horadam, A. F. (1994). Applications of modified Pell numbers to representations. Ulam Quarterly Journal, 3(1), 34-53.

[11] Johnson, R. C. (2009). Fibonacci numbers and matrices, Available online: https:// maths.dur.ac.uk/ dma0rcj/PED/fib.pdf. 
[12] Kalman, D. (1982). Generalized Fibonacci numbers by matrix methods. The Fibonacci Quarterly, 20(1), 73-76.

[13] Kilic, E. (2008). The Binet fomula, sums and representations of generalized Fibonacci p-numbers. European Journal of Combinatorics, 29(3), 701-711.

[14] Kilic, E., \& Tasci, D. (2006). The generalized Binet formula, representation and sums of the generalized order- $k$ Pell numbers. Taiwanese Journal of Mathematics, 10(6), 1661-1670.

[15] Kocer, E. G., \& Tuglu, N. (2007). The Binet formulas for the Pell and Pell-Lucas $p$-numbers. Ars Combinatoria, 85, 3-17.

[16] Koken, F., \& Bozkurt, D. (2008). On the Jacobsthal numbers by matrix methods. International Journal of Contemporary Mathematical Sciences, 3(13), 605-614.

[17] Lancaster, P. \& Tismenetsky, M. (1985). The Theory of Matrices: with Applications, Elsevier.

[18] Lidl, R., \& Niederreiter, H. (1994). Introduction to Finite Fields and Their Applications, Cambridge University Press.

[19] McDaniel, W. L. (1996). Triangular numbers in the Pell sequence. The Fibonacci Quarterly, 34(2), 105-107.

[20] Shannon, A. G., Anderson, P. G., \& Horadam, A. F. (2006). Properties of Cordonnier, Perrin and Van der Laan numbers. International Journal of Mathematical Education in Science and Technology, 37(7), 825-831.

[21] Shannon, A. G., Horadam, A. F., \& Anderson, P. G. (2006). The auxiliary equation associated with the plastic number, Notes on Number Theory and Discrete Mathematics, 12(1), 1-12.

[22] Stakhov, A. P. (1999). A generalization of the Fibonacci Q-matrix. Rep. Natl. Acad. Sci. Ukraine, 9, 46-49.

[23] Stakhov, A. P., \& Rozin, B. (2006). Theory of Binet formulas for Fibonacci and Lucas p-numbers. Chaos, Solitions, Fractals, 27(5), 1162-1177.

[24] Stewart, I. (1996). Tales of a neglected number. Scientific American, 274(6), 102-103.

[25] Tasci, D., \& Firengiz, M. C. (2010). Incomplete Fibonacci and Lucas p-numbers. Mathematical and Computer Modelling, 52(9-10), 1763-1770. 